\title{
Analysis of the Effects of SD Plasma on Aerodynamic Drag Reduction of a High-speed Train
}

\author{
Hyung-Woo Lee* and Hyeok-Bin Kwon ${ }^{\dagger}$
}

\begin{abstract}
Experimental analysis according to the plasma actuator design variables was performed in order to verify the effects of sliding discharge plasma on aerodynamic drag reduction of a high-speed train. For the study, sliding discharge plasma actuator and high-frequency, high-voltage power supply were developed and experimented to figure out the best design variables for highest ionic wind velocity which could reduce the drag force. And then, 5\% reduced-scale model of a high-speed train was built for wind tunnel test to verify it. From the results, it was confirmed that sliding discharge plasma had contribution to reduce the drag force and it had the potential to be applied to real-scale trains.
\end{abstract}

Keywords: Sliding discharge, SD plasma, Flow control, Aerodynamic drag, High-speed train

\section{Introduction}

As the speed of trains go higher and higher, the aerodynamic drag is one of key issues to be solved. This aerodynamic drag is known to be proportional to the square of train speed, and the required traction power is proportional to the third power of the train velocity as shown in Fig. 1 [1]. Therefore, the aerodynamic drag becomes dominant in a high speed operation. To increase the maximum speed of high-speed train as well as to reduce energy consumption to overcome resistance to motion, reducing aerodynamic drag of high-speed train is one of the most promising ways. Until now, passive flow controls by shape improvement or optimization on nose shape or adaption of fairings for inter-cars and bogie have generally been used to reduce the aerodynamic drag of high-speed train [2-4]. Active flow controls have also been suggested to overcome the limit of passive flow control and have widely been studied especially in aerospace and automotive industry. Among many active flow controls, plasma actuator is one of possible technologies [5-6].

Dielectric-barrier-discharge (DBD) is one of nonequilibrium discharges that can be operated at about atmospheric pressure and temperature, and are generally used for a number of applications such as ozone generator, excimer lamps, AC plasma display panels (PDP), and so on [7]. On the other hand, sliding discharge (SD), another non-equilibrium discharge, produces wider area of the plasma and more induced air flow than DBD. Therefore, SD might be one of good candidates to apply to the aerodynamic drag reduction while DBD plasma actuator is

$\dagger$ Corresponding Author: Department of Transportation System Engineering, Korea National University of Transportation, Korea. (hbkwon@ut.ac.kr)

* Department of Railway Vehicle System Engineering, Korea National University of Transportation, Korea. (krhwlee@ut.ac.kr) Received: January 8, 2014; Accepted: April 29, 2014

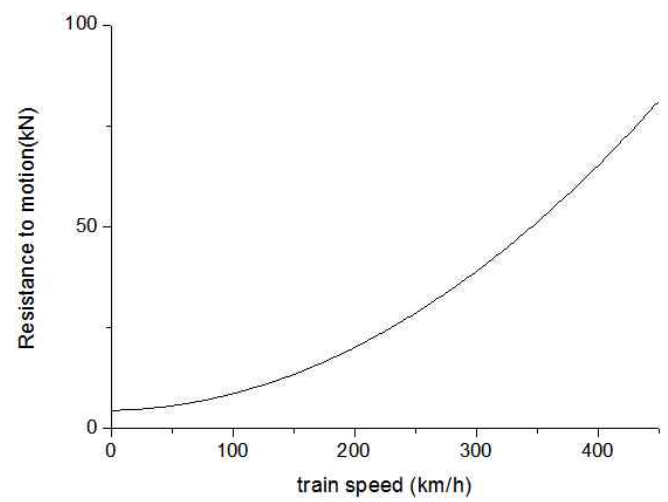

Fig. 1. Aerodynamic drag according to the velocity [1]

widely used. Even though flow control to reduce the aerodynamic drag by using SD plasma has been developed recently, insufficient data of performance variables of the SD plasma make it difficult to predict the system performance. Therefore experimental analysis according to the actuator design variables should be followed.

Many studies about air flow control have been made to alleviate problems such as increase in fuel consumption of the aircraft, mental damage caused by the aircraft noise, speed limits of high-speed trains due to the drastic increase of aerodynamic drag.

There are passive and active flow controls to reduce aerodynamic drag. Passive methods have been studied for a long time such as shape improvement or optimization on nose, or adaption of fairings for inter-cars and bogie of the train. However, it is getting difficult to expect any more effectiveness as trains run higher speed. Among various flow control methods as shown in Fig. 2, most common way of the active flow controls is air injection or suction by using mechanical devices. Recently, flow control using plasma actuators have been actively studied because plasma actuator has a simple structure, ease of installation, 


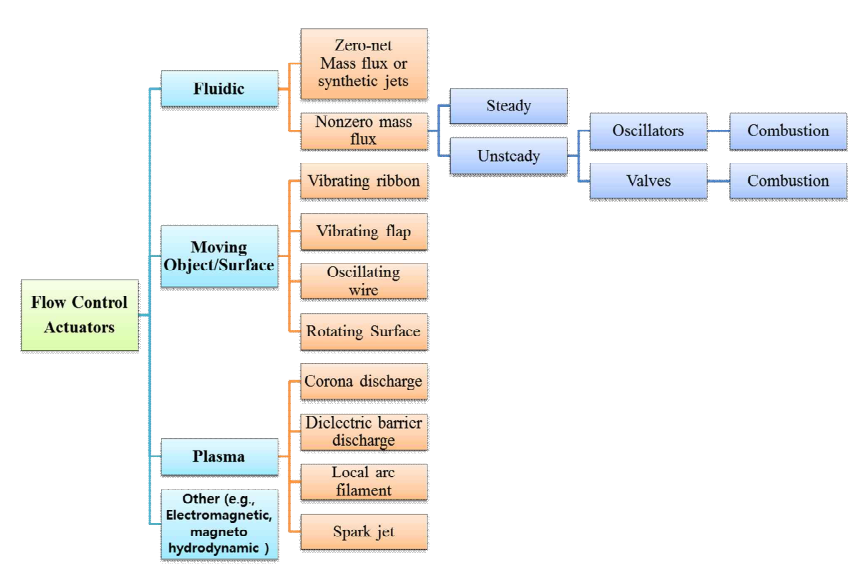

Fig. 2. Flow control actuators

taking less bulky, and fast response time.

A study on the use of plasma actuators to reduce aerodynamic drag was conducted in Korea, 2012 [8]. This study mainly focuses on the DBD actuator to control the ionic wind. The ionic wind means a flow of ionized fluid, which is created by strong electric field. The ionic wind is also called an ion wind, coronal wind or electric wind. Velocity of the ionic wind is around several $\mathrm{m} / \mathrm{s}$ [9]. The drag reduction effect of the DBD actuator has also been examined by wind tunnel experiment using 2-dimensional wedge model [10] and the deduced drag reduction rate was $9.7 \%$ under free stream wind velocity of $2 \mathrm{~m} / \mathrm{s}$, however, the drag reduction rate decreased as increasing the wind velocity and the effect of drag reduction was not appeared over $20 \mathrm{~m} / \mathrm{s}$. In 2010, Y. Li, et al. had successful experiment to reduce the aircraft noise by using DBD and SD plasma actuators [11]. However, the SD plasma actuator has not been used for aerodynamic drag reduction so far.

In this paper, SD plasma actuator and high voltage power supply were developed and experimented in order to figure out the best design variables for highest ionic wind velocity and small-scale physical mock-up of a high-speed train was built and wind tunnel test was performed to verify the aerodynamic drag reduction.

\section{SD Plasma and Experimental Equipment for Ionic Wind}

The plasma actuator is classified into Sliding Discharge (SD) type and Dielectric Barrier Discharge (DBD) type by structure of electrodes as shown in Fig. 3. And the basic discharge method to make plasma is Barrier discharge. Barrier discharge is generating gas discharge when alternating current (AC) voltage is applied between a pair of electrodes, which are with a dielectric not to occur direct discharge on electrodes. It is also called silent discharge.

The Actuator which uses only barrier discharge is DBD type. DBD type has a dielectric between two electrodes. When AC source is applied to electrodes, plasma is

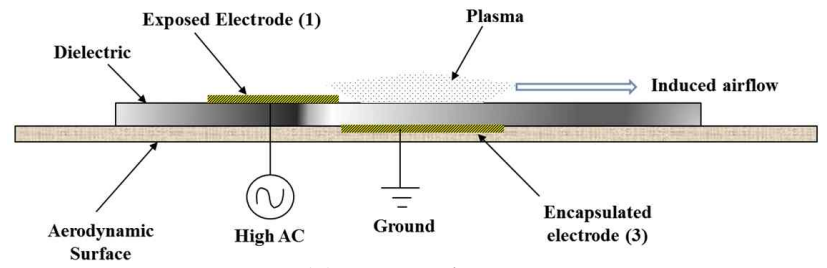

(a) DBD Plasma

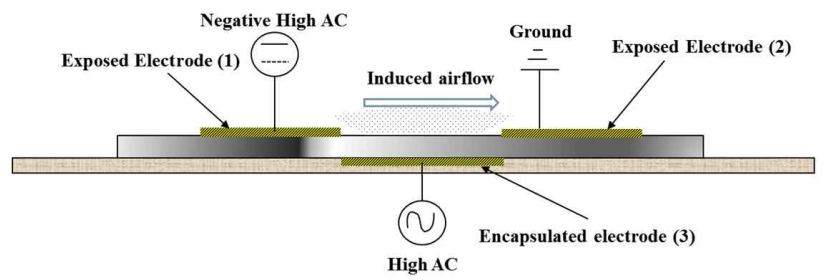

(b) SD Plasma

Fig. 3. Configuration of the DBD and SD plasma actuators

generated and ionic wind is also generated by it. Direction of ionic wind is from bare electrode to wrapped electrode, horizontal direction.

SD type uses barrier discharge and corona discharge. It is consist of three electrodes, one electrode is located a side of dielectric and others are located the opposite side of dielectric as shown in Fig. 3(b). Both AC source and DC source are used. The sequence of applying electric power is that AC voltage is applied firstly and then DC voltage is applied. Ionic wind in vertical direction is generated by $\mathrm{AC}$ voltage and ionic wind in horizontal direction is generated by DC voltage. So, we can control both of vertical and horizontal direction by using only SD. In vertical direction ionic wind, DBD type has an advantage that its simple structure over SD type. But SD type has a fast flow velocity, more stable generation of plasma and controllability of both vertical and horizontal direction. For the reasons mentioned above, it can be said that SD type is better than DBD type for flow control.

For the actuator, the mechanism of flow induced by body force vector field that couples with the momentum in the external flow is considered. Although it is considered useful only at low speeds, SD can be effective at high speeds by the optimized actuator designs which can be developed through better understanding and modeling of the actuator physics. In this study, optimal design of the SD plasma actuator to generate sufficient air flow (ionic wind) has been developed so as to control turbulent flow and reduce the surface resistance.

SD plasma for flow control has very different performances according to the power supply, frequency and configuration of electrodes such as height, width, spacing, arrangement, and so on. In this study, experiment of the ionic wind which is generated by the SD plasma according to variable design parameters has been done prior to the wind tunnel test. Table 1 shows the variables used to figure out the SD mechanism and ionic wind. 
Table 1. System variables used to figure out the mechanism between SD plasma and ionic wind

\begin{tabular}{c|c}
\hline Variables & \\
\hline Input AC voltage waveform & Quasi-square waveform \\
Input AC voltage magnitude & $1-4[\mathrm{kV}]$ \\
Input AC voltage frequency & $10-40[\mathrm{kHz}]$ \\
Input DC voltage magnitude & $10-25[\mathrm{kV}]$ \\
Distance between electrodes & $10-20[\mathrm{~mm}]$ \\
Electrodes width & $0.075[\mathrm{~mm}]$ \\
Electrodes length & $100[\mathrm{~mm}]$ \\
Dielectric & $0.125[\mathrm{~mm}]$, Kapton \\
\hline
\end{tabular}

\subsection{Power supply for SD plasma actuator}

For operation of plasma actuator, both high-frequency high-voltage AC source and high-voltage DC source are required.

To get the high-voltage high-frequency AC source, an inverter and a transformer is used. Because of high frequency input voltage, a general transformer cannot be used, but a high-frequency transformer. Compared with a general transformer, a high-frequency transformer can be miniaturized and light by increasing available frequency.

To get the high-voltage DC source, the Cockcroft-Walton (CW) circuit is used that gains DC high-voltage from $\mathrm{AC}$ input voltage. The $\mathrm{CW}$ has an advantage of easy boost voltage by adding more ladders. This one ladder consists of two capacitors and two diodes and each can boost voltage twice. But, of course, it has limitation of voltage boosting as the number of ladder increases.

In this study, single-phase half bridge inverter circuit was used for high-frequency. As the number of switch is fewer than full bridge, the single-phase half bridge circuit

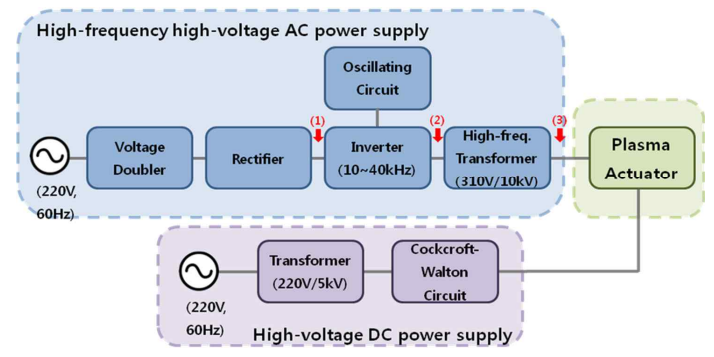

(a) Configuration of the power supply

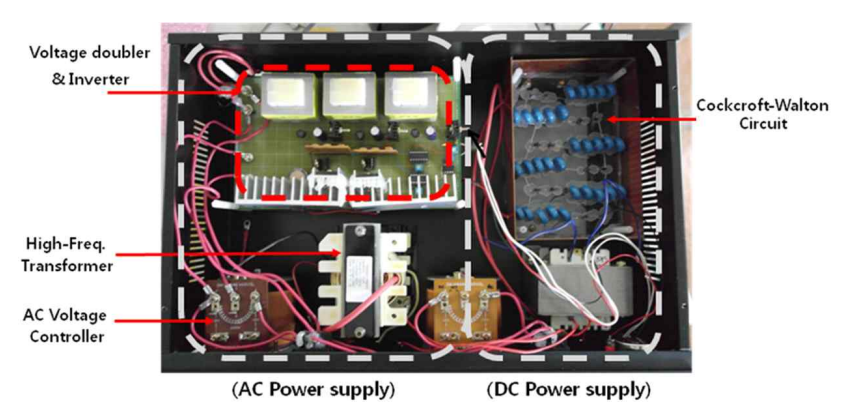

(b) Real picture of the power supply

Fig. 4. Configuration and real picture of the power supply for SD plasma actuator

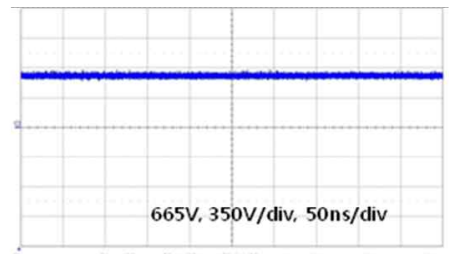

(a) Waveform after rectifier, (1) in Fig. 4(a)

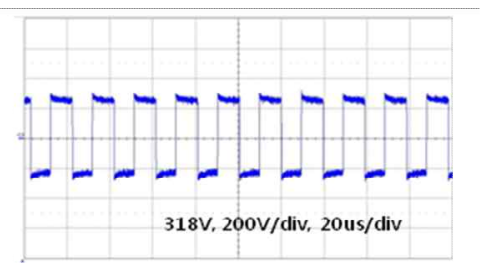

(b) Waveform after inverter, (2) in Fig. 4(a)

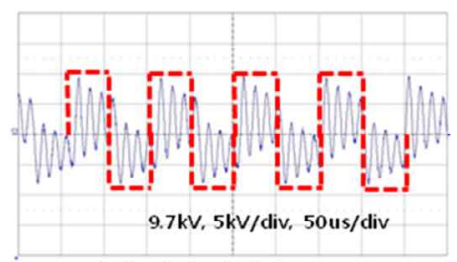

(c) Waveform after HF transformer, (3) in Fig. 4(a)

Fig. 5. Output waveform of the power supply for SD plasma actuator

has more simple structure and convenient switch control. And high-frequency transformer was also used for AC high-voltage. The high-frequency transformer boosts voltage from $320 \mathrm{~V}$ to $10 \mathrm{kV}$ and was made to use a range of 10 $\mathrm{kHz}$ to $40 \mathrm{kHz}$. To get the $\mathrm{DC}$ high-voltage, the $\mathrm{CW}$ circuit was used as mentioned before. But owing to limitation of voltage boost, an initial input voltage was boosted up to 5 $\mathrm{kV}$ by a transformer and it had six ladders, therefore the maximum output voltage of the DC power was $28 \mathrm{kV}$. Figs. 4 and 5 show the power supply which was built for the experiment and output waveform of the power supply for SD plasma actuator, respectively.

\subsection{SD plasma actuator}

As shown in Fig. 3, SD plasma actuator consists of substrate (base plate), dielectric and three electrodes such as high-frequency high-voltage $\mathrm{AC}$ electrode, high-voltage DC electrode and ground electrode. The substrate was made of flexible polyester to mount on the small-scale mock-up model of a high-speed train for wind tunnel test. The dielectric was made by Kapton which was a polyimide film which could remain stable in a wide range of temperatures. The thickness of the Kapton was $0.125 \mathrm{~mm}$ including adhesive in order to apply high-voltage AC. The $0.07 \mathrm{~mm} \mathrm{3M}$ conductive copper tape was used for the electrode and the distance between electrodes are $10 \mathrm{~mm}$, $15 \mathrm{~mm}$ and $20 \mathrm{~mm}$, respectively appeared in Fig. 6 . 


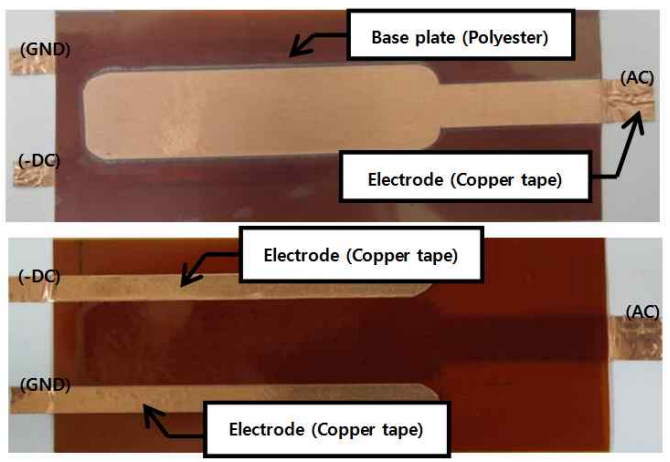

(a) Front and rear view of the SD plasma actuator

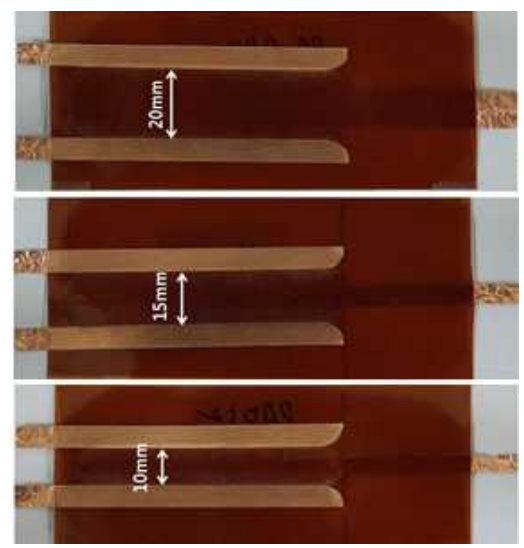

(b) Distance between electrodes built

Fig. 6. SD plasma actuators

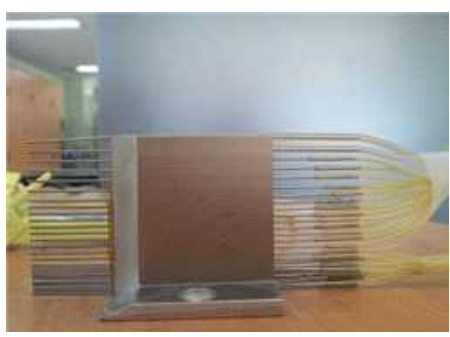

(a) Rake array

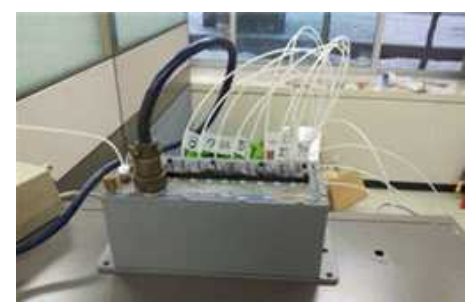

(b) Multi-channel pressure scanner

Fig. 7. Ionic wind measurement system

\subsection{Ionic wind measurement}

Performance of the SD plasma was evaluated by measuring ionic wind on the rear of the actuator using multi-channel pressure measurement as in Fig. 7.
The probe was installed back $0 \mathrm{~mm}$ (X-axis) away from the actuator and the ionic wind was measured up to $18 \mathrm{~mm}$ in vertical direction (Y-axis) based on the bottom. In order to find out maximum induced air flow, experiments of the ionic wind according to the $\mathrm{AC}$ voltage frequency, $\mathrm{AC}$ voltage magnitude, DC voltage magnitude, and electrode spacing, were put in progress in the number of cases for a total of 192 .

\section{Experimental Results}

\subsection{Experimental results of SD plasma}

In 192 cases, several significant cases are represented as follows. Fig. 8 shows the ionic wind varying DC voltage from $10 \mathrm{kV}$ to $25 \mathrm{kV}$ in the condition of $20 \mathrm{~mm}$ electrode spacing, $\mathrm{AC} 25 \mathrm{kHz}$ and $3 \mathrm{kV}$. In the graph, the peak velocity of ionic wind seems to get larger with increasing DC voltage.

Fig. 9 shows the ionic wind varying $\mathrm{AC}$ voltage from $2.31 \mathrm{kV}$ to $3.98 \mathrm{kV}$ in the condition of $\mathrm{AC} 15 \mathrm{kHz}$ frequency, $20 \mathrm{~mm}$ electrode spacing and DC $10 \mathrm{kV}$. Similar to the case of DC voltage variation experiment in Fig. 8, the ionic wind get stronger with increasing voltage except

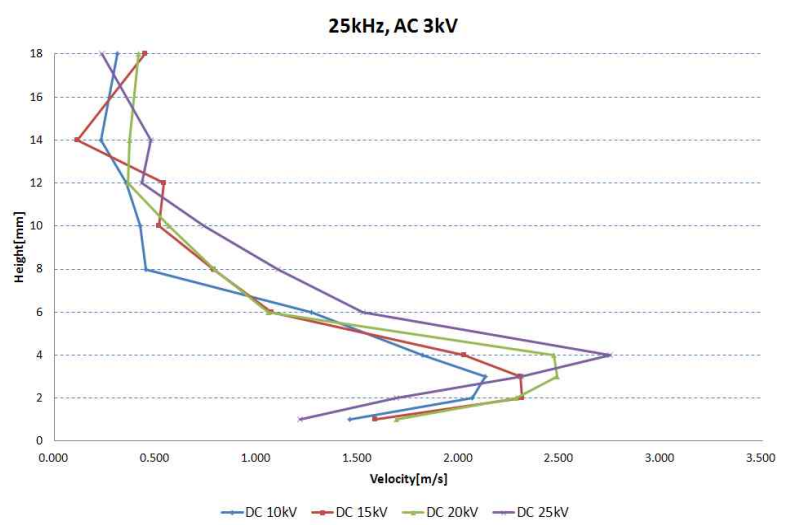

Fig. 8. Ionic wind velocity measured varying DC voltage magnitude

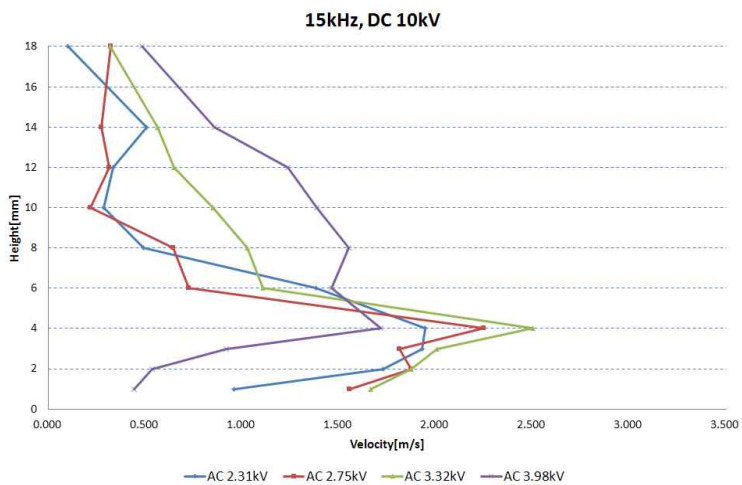

Fig. 9. Ionic wind velocity measured varying AC voltage magnitude 


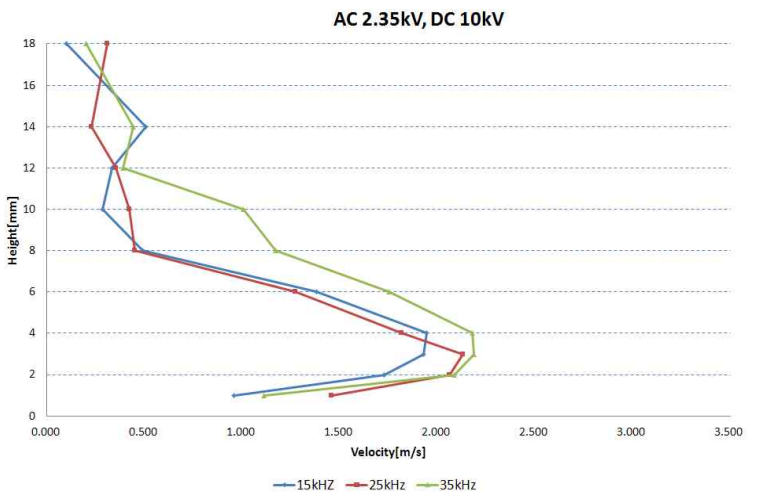

Fig. 10. Ionic wind velocity measured varying AC voltage frequency

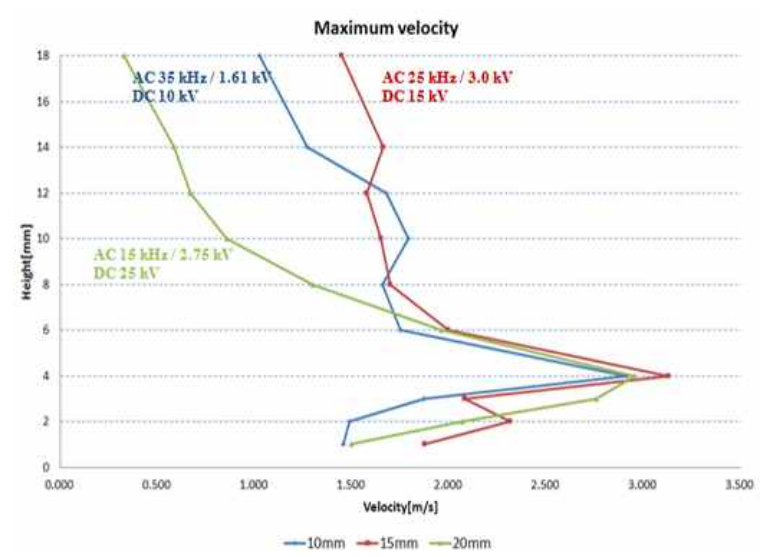

Fig. 11. Maximum ionic wind velocity measured varying electrode spacing

at the maximum $\mathrm{AC}$ voltage $3.98 \mathrm{kV}$ which yield the minimum ionic wind intensity. The higher excitation voltage leads to stronger discharge of plasma, however, too high excitation voltage can cause reverse plasma discharge [8]. In case of $\mathrm{AC}$ voltage variation experiment, such reverse plasma discharge could be used to describe the saturation of ionic wind intensity versus excitation $\mathrm{AC}$ voltage.

Fig. 10 shows the ionic wind varying $\mathrm{AC}$ voltage frequency from $15 \mathrm{kHz}$ to $35 \mathrm{kHz}$ in the condition of $\mathrm{AC}$ $2.35 \mathrm{kV}, 20 \mathrm{~mm}$ electrode spacing and DC $10 \mathrm{kV}$. The peak wind velocity of ionic wind seems to be proportional to the frequency which agrees with the previous study by DBD plasma [8].

Fig. 11 shows the ionic wind varying electrode spacing from $10 \mathrm{~mm}$ to $20 \mathrm{~mm}$ in the different condition of power supply to get maximum air flow respectively. It was deduced that proper electrode spacing can be derived by varying the $\mathrm{DC} / \mathrm{AC}$ voltage and frequency for stronger ionic wind. From the experimental results, it was known that the performance of the SD plasma actuators was influenced by many system parameters and the maximum ionic wind was generated in the condition of $15 \mathrm{~mm}$ electrode spacing, DC $15 \mathrm{kV}$, AC $25 \mathrm{kHz}$ and $3 \mathrm{kV}$.

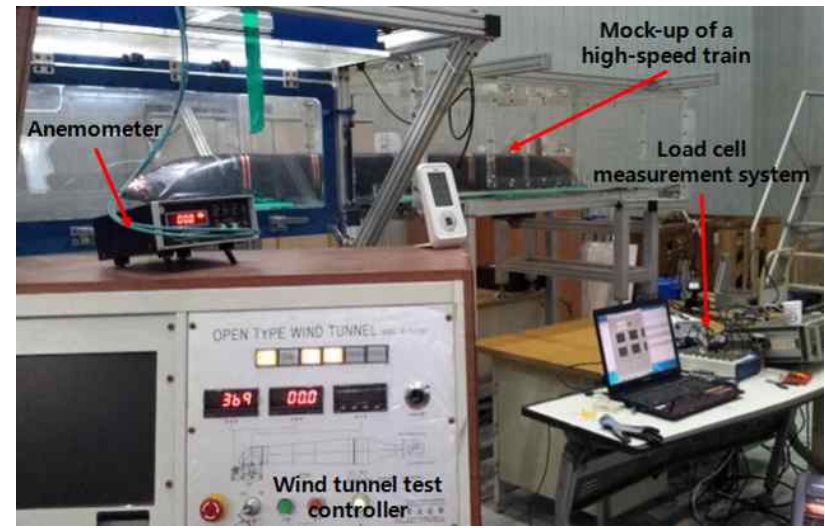

Fig. 12. Equipment of the wind tunnel test
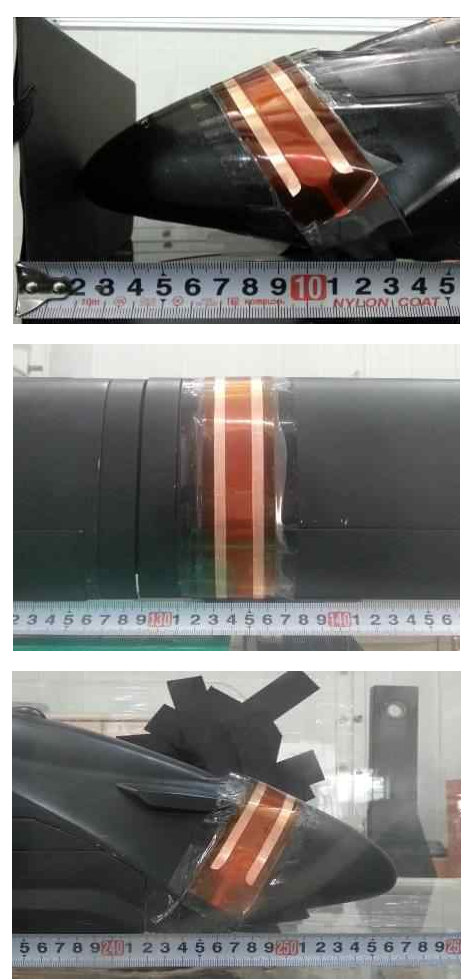

Fig. 13. SD plasma actuators which are mounted on front, middle and rear parts of the mock-up model (700 $\mathrm{mm}, 1320 \mathrm{~mm}$ and $2440 \mathrm{~mm}$ away from the train nose, respectively)

\subsection{Experimental results of aerodynamic drag}

$1 / 20^{\text {th }}$ reduced scale model of a high-speed train was built and wind tunnel test was performed to verify the effect of SD plasma actuator on the aerodynamic drag reduction. The optimal SD plasma actuator found from the experiment was mounted on a mockup of a high-speed train in Korea Railroad Research Institute and the performance of the SD plasma was evaluated by measuring ionic wind on the rear of the actuator using Hot-wire anemometer and by measuring total drag force using load cell as shown in Fig. 12. The speed of the wind tunnel test 
was $20 \mathrm{~m} / \mathrm{s}$ by the limitation of the facility.

SD plasma actuator used for wind tunnel test was 15 mm electrode spacing, DC $15 \mathrm{kV}$ and AC $25 \mathrm{kHz}, 3 \mathrm{kV}$ power supply system. Fig. 13 shows the SD plasma actuators which were mounted and measured on front, middle and rear parts of the mock-up model by considering interference with the load cell measurement equipment (700 $\mathrm{mm}, 1320 \mathrm{~mm}$ and $2440 \mathrm{~mm}$ away from the train nose, respectively).

From the experimental results, the aerodynamic drag was reduced by $0.55 \%$ in $10 \mathrm{~m} / \mathrm{s}$ and $1.86 \%$ in $20 \mathrm{~m} / \mathrm{s}$ (from $3.041 \mathrm{~N}$ to $2.986 \mathrm{~N}$ ), respectively. These were very meaningful results considering the streamline shape of the model train which had small flow separation at the trailing part of the train.

\section{Conclusion}

SD plasma actuator to reduce the aerodynamic drag of a high-speed train was introduced in this paper. It was about discharge physics and the plasma chemistry involved on one side and high-voltage power electronics on second side, and aerodynamics on third side. As insufficient data of performance variables of the SD plasma made it difficult to predict the system performance and to apply this technique on trains, experimental analysis according to the actuator design variables in order to verify the effects of SD plasma on drag reduction, was performed in this study. SD plasma actuator and high voltage power supply were built and experimented in order to figure out the best design variables for biggest ionic wind and then small-scale physical mockup of a high-speed train was built and wind tunnel test had verified the effect of SD plasma on aerodynamic drag reduction. There is no doubt that the described application of sliding discharge will continue to grow and emerge to commercial trains in the near future, and further study is needed in order to improve its effectiveness.

\section{Acknowledgements}

This work was supported by a grant from the academic research program of Korea National University of Transportation in 2013 .

\section{References}

[1] H. Kwon, S. Yun and H. Lee, "Target and Implementation of Aerodynamic Drag Reduction for Highspeed Train to Reach Up to $500 \mathrm{~km} / \mathrm{h}$ Running Speed", Proc. of KSR fall conference, 2011

[2] J. L. Peters, "Aerodynamics of high speed trains and Maglev vehicles”, Int. J. of Vehicle Design, SP3, pp. 308, 1993
[3] A. P. Gaylard, A. B. Howlett and D. J. Harrison, "Assessing Drag Reduction Measures for High-speed Train”, The Royal Aeronautical Society, 1994

[4] L. Gori, G. Pugi, M. T. Cambini, A. G. Violi, "Acoustic characterisation of high speed train ETR 500", Proc. of 2001 WCRR Conference, 2001

[5] M. Forte, J. Jolibois, et. al., "Optimization of a dielectric barrier discharge actuator by stationary and non stationary measurements of the induced flow velocity application to airflow control", Experiments. Fluids, pp. 917-928, 2007

[6] T. Corke, C. Enloe and S. Wilkinson, "Dielectric barrier discharge plasma actuators for flow control", Annual reviews Fluid Mechanics, Vol. 43, pp. 505529,2010

[7] U. Kogelschatz, B. Eliasson and W. Egli, "Dielectric barrier discharges principle and applications", Journal de Physique IV, Vol. C4, pp. 47-66, 1997

[8] S. H. Yun, H. B. Kwon, and T. Kim, "Study on characteristics of DBD plasma actuator as design parameters for plasma flow control", J. of KSAS, Vol. 40, pp. 492, 2012

[9] M. Robinson, "A history of the electric wind", American J. of Physics, Vol. 30, pp. 366, 1962

[10] S. H. Yun and T. Kim, "Flow Separation Control on 2-D Bluff Body using Dielectric Barrier Discharge Plasma Actuators", Proc. of 2012 Asia-Pacific Int. Sym. on Aerospace Technology, 2012

[11] Yong Li, X. Zhang and X. Huang, "The use of plasma actuators for bluff body broadband noise control”, Experiments Fluids, Vol. 49, pp 367, 2010

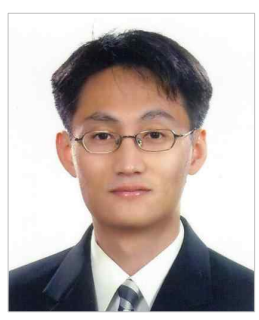

Hyung-Woo Lee He received the B.S. and M.S. degrees from Hanyang University, Seoul, Korea, in 1998 and 2000, respectively, and the Ph.D. degree from Texas A\&M University, College Station, TX, in 2003, all in electrical engineering. In 2004, he was a Post-doctoral Research Assistant in the Department of Theoretical and Applied Mechanics, Cornell University, Ithaca, NY. In 2005, he was a contract Professor at the BK division of Hanyang University, Seoul, Korea. From 2006 to 2012, he worked as a senior researcher at the Korea Railroad Research Institute, Uiwang, Korea. He joined Korea National University of Transportation as professor of the department of Railway Vehicle System Engineering in March 2013. His research interests include design, analysis and control of motor/generator, power conversion systems, and applications of motor drives such as Maglev trains, conventional railway propulsion systems, and modern renewable energy systems. 


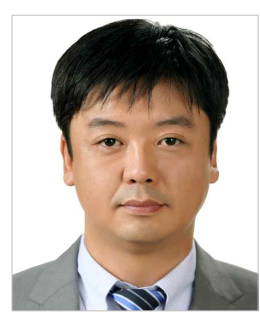

Hyeok-Bin Kwon $\mathrm{He}$ received the B.S., M.S. and Ph.D degrees from Seoul National University, Seoul, Korea, in 1995, 1997 and 2001, respectively, all in aerospace engineering. From 2001 to 2013, he worked as a senior researcher and principal researcher at the Korea Railroad Research Institute,

Uiwang, Korea. He joined Korea National University of Transportation as professor of the department of Transportation System Engineering in October 2013. His main research interests are aerodynamics of high-speed train including and related topics including aerodynamic drag reduction, train-tunnel interaction, crosswind safety and ballast-flying problem. 